\title{
Robust Trapdoor Tarantula Haploclastus validus Pocock, 1899: notes on taxonomy, distribution and natural history (Araneae: Theraphosidae: Thrigmopoeinae)
}

\author{
Zeeshan A. Mirza ${ }^{1}$, Rajesh V. Sanap ${ }^{2} \&$ Manju Siliwal ${ }^{3}$ \\ ${ }^{1}$ Zoology Department, Bhavans College, Andheri (W), Mumbai, Maharashtra 400058, India \\ 2 D5/2, Marol Police Camp, Marol Maroshi Road, Andheri (E), Mumbai, Maharashtra 400059, India \\ ${ }^{3}$ Wildlife Information Liaison Development Society, 9-A, Lal Bahadur Colony, Peelamedu, Coimbatore, Tamil Nadu 641004, India \\ Email: ${ }^{1}$ snakeszeeshan@gmail.com (corresponding author), ${ }^{2}$ rajeshvsanap@gmail.com, ${ }^{3}$ manjusiliwal@gmail.com
}

\section{Date of publication (online): 26 October 2011 Date of publication (print): 26 October 2011 ISSN 0974-7907 (online) | 0974-7893 (print) \\ Editor: Ansie Dippenaar-Schoeman \\ Manuscript details: \\ Ms \# 02627 \\ Received 15 November 2010 \\ Final received 29 August 2011 \\ Finally accepted 23 September 2011}

Citation: Mirza, Z.A., R.V. Sanap \& M. Siliwa (2011). Robust Trapdoor Tarantula Haploclastus validus Pocock, 1899: notes on taxonomy, distribution and natural history (Araneae: Theraphosidae: Thrigmopoeinae). Journal of Threatened Taxa 3(10): 2109-2119.

Copyright: $\odot$ Zeeshan A. Mirza, Rajesh V. Sanap \& Manju Siliwal 2011. Creative Commons Attribution 3.0 Unported License. JoTT allows unrestricted use of this article in any medium for non-profit purposes, reproduction and distribution by providing adequate credit to the authors and the source of publication.

Author Contribution: ZM \& RS carried out surveys at Aarey Milk Colony and Matheran. MS carried out surveys at Bhimashankar Wildlife Sanctuary. The paper received equal contribution from all the authors.

For Author Details \& Acknowledgements see end of this article

ation bir

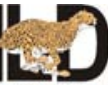

AUNA \& FLORA INTERNATIONAL
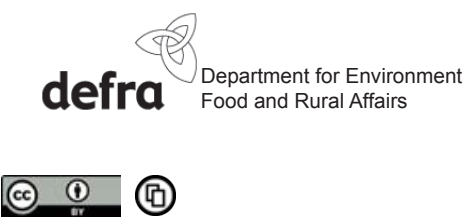

OPEN ACCESS | FREE DOWNLOAD
Abstract: The genus Haploclastus is endemic to India and is represented by six species. One of the species $H$. validus Pocock, 1899 was described from Matheran and has remained poorly known in terms of its natural history and distribution. During recent surveys the species was for the first time found again since its description nearly 110 years ago. Based on the new material collected it is redescribed and data on its natural history and distribution are added. It is the first record of an Indian theraphosid spider, which closes its burrow with a trapdoor.

Keywords: Haploclastus validus, taxonomy, Theraphosidae, trapdoor spider, Western Ghats.

\section{INTRODUCTION}

The genus Haploclastus is endemic to India and is represented by six species, namely, Haploclastus cervinus Simon, 1892, H. kayi Gravely, 1915, H. nilgirinus Pocock, 1899, H. satyanus Barman, 1978, H. tenebrosus Gravely, 1935 and H. validus Pocock, 1899 (Siliwal et al. 2005; Siliwal \& Raven 2010; Platnick 2011) of which five have been reported from the Western Ghats (Pocock 1900; Gravely 1915, 1935; Molur \& Siliwal 2004). While conducting surveys in Aarey Milk Colony, Mumbai, authors (RS \& ZM) collected specimens of both sexes of the genus Haploclastus. Initially, it was considered a trapdoor spider of the family Ctenizidae due to its trapdoor burrow structure and some morphological characters. After examining the specimens under a stereomicroscope, it was identified as belonging to the genus Haploclastus of the family Theraphosidae based on the presence of a distinct maxillary heel, the apical segments of the PLS being digitiform, the presence of claw tufts and absence of a rastellum (Dippenaar-Schoeman 2002). Members of the genus Haploclastus possess numerous horizontally aligned thorn-like setae arranged in two to three rows above and below the maxillary suture along with long tapering modified setae aligned vertically in a diffuse pattern on the prolateral side of the maxilla which are distinctly present in females. The species of Haploclastus, which had the closest distribution range to Mumbai, was H. validus from Matheran. To confirm this, surveys were carried out in

Abbreviations: ALE - Anterior lateral eye; AMC - Aarey Milk Colony; AME - Anterior median eye; $d$ - dorsal; fe - femur; me - metatarsus; MOQ - Median ocular quadrate; MS - Manju Siliwal; p - prolateral; pa - patella; PLE - Posterior lateral eye; PLS - Posterior lateral spinnerets; PME - Posterior median eye; PMS - Posterior median spinnerets; $r$ retrolateral; ta - tarsus; ti-tibia; v - ventral. 
Matheran, Raighad District, Maharashtra, the type locality of $H$. validus. From Matheran, both male and female specimens belonging to a single Haploclastus species were collected. This confirms that only one species of Haploclastus occurs in Matheran. Moreover, after examining male and female specimens from Matheran, it was clear that the recent synonym of $H$. robustus with $H$. validus by Siliwal \& Raven (2010) was valid. In addition to this, females and males from both the localities (Matheran and Aarey Milk Colony) shared characters that were found to be common, further supporting that the species from AMC was $H$. validus.

According to Siliwal \& Raven (2010), the type specimens of $H$. validus is presumed to be either lost or deposited in some European museum. Therefore, redescription of this species based on type specimens is not possible. The original description of the $H$. validus lacks information on the species natural history, detailed morphometry of legs and structure of genitilia. Molur \& Siliwal (2004) coined a common name for this species as 'Strong Large Burrowing Spider' which needs to be changed as this species is now known to be a trapdoor spider and hence, we propose it as 'Robust Trapdoor Tarantula'.

\section{MATERIAL AND METHODS}

Specimens were collected during opportunistic surveys in different parts of Maharashtra. Measurements were taken with a Mitutoyo ${ }^{\mathrm{TM}}$ Dial Caliper. All measurements are in $\mathrm{mm}$. Spermathecae were dissected and cleared in clove oil. Total length excludes chelicerae. All illustrations were prepared with the help of camera lucida by MS and morphological details were observed under Labomed stereo-binocular microscope. Descriptive style follows the standardized descriptive style provided by Siliwal $\&$ Molur $(2007,2009)$. All specimens are deposited at the Wildlife Information Liaison Development Society (WILD), Coimbatore, Tamil Nadu, India.

\section{Taxonomy}

\section{Haploclastus Simon, 1892}

Phlogiodes Pocock 1899: 748; Pocock 1900: 179; Smith 1987: 94.

Haploclastus Simon, 1892: 269; Raven 1985:157
Type species: Haploclastus validus Pocock, 1899

Diagnosis: Fovea deep and slightly procurved; numerous horizontally aligned thorn-like setae in two to three rows above and below the maxillary suture along with long tapering modified setae aligned vertically in diffuse pattern on prolateral side of maxilla (Raven 1985).

\section{Haploclastus validus Pocock, 1899}

Haploclastus robustus Pocock, 1899: 748; Haploclastus validus, Siliwal \& Raven 2010: 72

Type material: Holotype female of $H$. validus (= H. robustus), Matheran, Raighad District, Maharashtra, coll. Bombay Natural History Society, deposited at Natural History Museum, London. Not examined.

\section{Material examined}

1 male, 27.iv.2010, Matheran, Raighad District, Maharashtra $\left(19^{\circ} 00^{\prime} \mathrm{N} \& 7^{\circ} 17^{\prime} \mathrm{E}\right.$; $800 \mathrm{~m}$ elevation), coll. Rajesh Sanap and Zeeshan Mirza, WILD-10ARA-1102; 1 female, 19.ii.2010, Matheran, Raighad District, Maharashtra $\left(19^{\circ} 00^{\prime} \mathrm{N} \& 7^{\circ} 17^{\prime} \mathrm{E} ; 800 \mathrm{~m}\right.$ elevation) coll. Ashish Jadhav \& Rajesh Sanap WILD10-ARA-1103; 1 male, 24.vi.2009, Aarey Milk Colony, Mumbai, Maharashtra (1907'31'N \& 72052'76"E; $104 \mathrm{~m}$ elevation), coll. Zeeshan Mirza \& V. Rathode, WILD-10-ARA-543; 1 female, 28.xii.2009, Aarey Milk Colony, Mumbai, Maharashtra, (1907'31'N \& 72052'76"E; 104m elevation), coll. Rajesh Sanap \& Zeeshan Mirza, WILD-10-ARA-544; 1 female, Bhimashankar WLS, Maharashtra, (19004'04.4"N \& 73'32'20.3”'E; 858m elevation), coll. Manju Siliwal, Saroj Behera \& Mandar Kulkarni, WILD-09ARA-360.

\section{Diagnosis}

Male - Carapace slightly shorter than metatarsus + tibia of leg I; slightly longer than patella + femur of leg III; considerably shorter than tarsus + metatarsus of leg IV and tibia + patella of leg I. ALE clearly larger than the rest. Embolus broad at base, slightly twisted anteriorly and flatten, flattened end resembles a duck's bill which tapers into a stout point; ventrally with rows of poorly developed keels.

\section{Female}

Carapace slightly longer than metatarsus + tibia of leg IV; slightly shorter than twice the length of tarsus + 
metatarsus of leg II. Fused seminal receptacle; brickshaped structure with the upper edges smooth and rounded rose to form small mounds.

\section{Redescription of male (Figs 1-11), WILD-10- ARA-1102}

Total length 26.10; carapace 12.46 long, 9.10 wide; abdomen 13.64 long and 9.10 wide, chelicerae 6.96 long. Spinnerets: PMS, 1.70 long, 0.60 wide, 0.84 apart; PLS, 6.82 total length (2.58 basal, 1.80 middle, 2.44 distal; midwidths1.12, 0.86, 0.62 respectively). Morphometry of leg and palp given in Table 1.

Colour in life (Image 1): Carapace blackish-brown, carapace overall covered with dense silvery curved grey hair radiating from fovea. Abdomen covered with thick mat of brownish-black hair. Femora, patellae and tarsi of all legs covered with brownish hair; metatarsus and tibia of all legs covered with silvery grey hair.

Carapace (Fig. 1): Fovea procurved. Length to width 1.6 , bristles: 14 long on caput in mid-dorsal line; 9 long, 6 short anteromedially; 14 long, 12 short between PME; 3 short between AME-AME; 7 long, 4 short on clypeal edge.

Eyes (Fig. 2): Ocular group 0.86 long, 2.14 wide; diameter AME 0.40, PME 0.18, ALE 0.46, PLE 0.30; distance between ALE-AME 0.21, AME-AME 0.26, PLE-PME 0.12, PME-PME 1.10; MOQ not square, 0.42 long, 0.86 front width, 1.34 back width.

Maxillae (Figs 3 \& 6): Prolateral face with randomly arranged long bristles above and below maxillary suture. Cuspules ca. 200 in triangular patch in anterior corner. 3.82 front length, 4.68 back length, 2.32 wide.

Maxillary lyra: No definite shape, small spike setae above and below suture arranged randomly. No ventral brush of lyra.

Labium (Fig. 3): 1.45 long, 1.76 wide with ca. 220 cuspules restricted to upper region of labium covering $1 / 4$ of area.

Chelicerae (Figs 4 \& 5): Ectal lyrate, grove glaborous; lyra, straight, black spines in 3-4 rows. Promarginal teeth in 2 rows: 11 outer large teeth, 6 small on inner edge adjacent to larger teeth; 50 basomesal teeth in 3-4 rows.

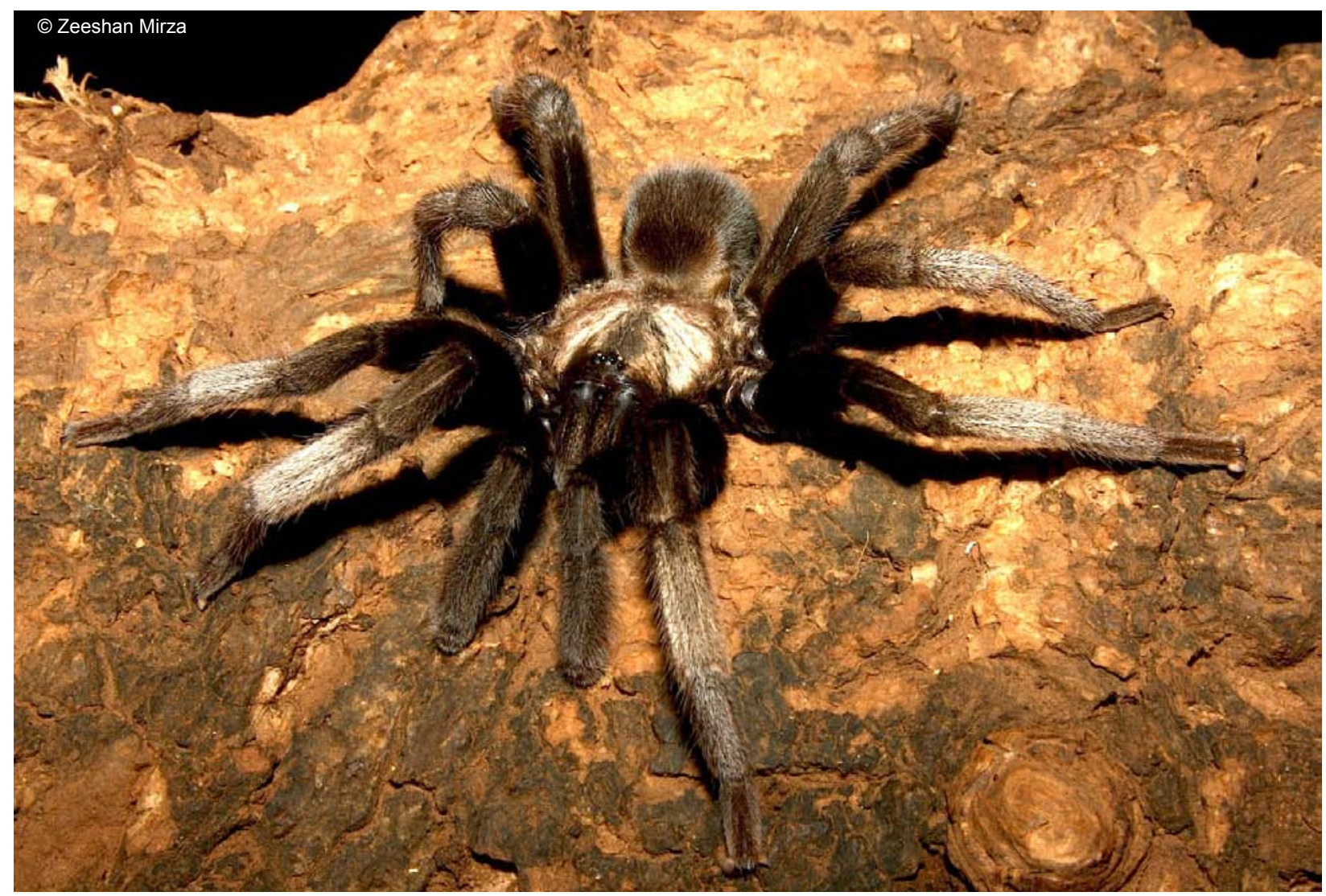

Image 1. Haploclastus validus male from Matheran (Raighad District, Maharashtra) 

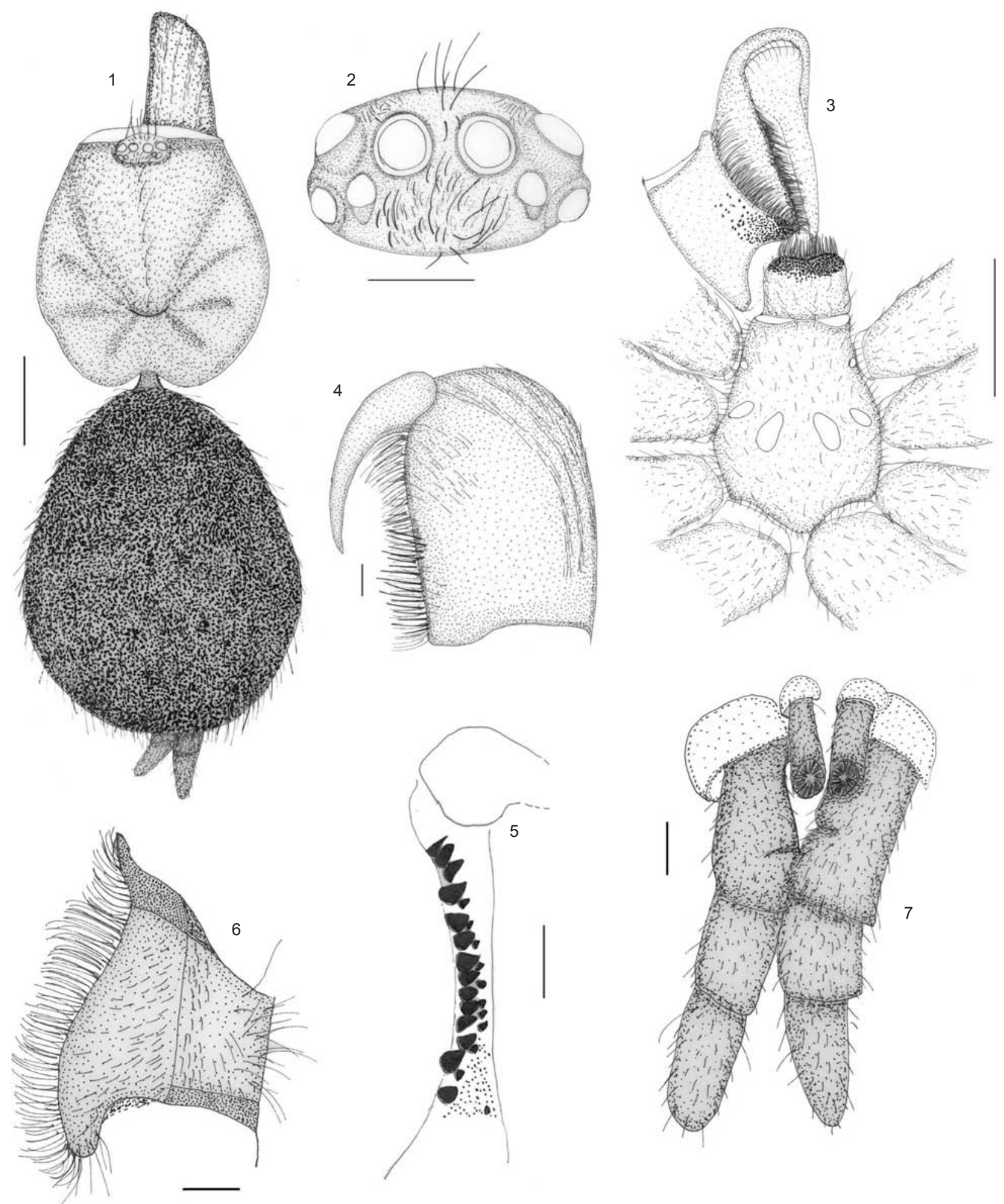

Figures 1-7. 1 - Dorsal view of spider, scale 0.5mm; 2 - Eye, scale 1mm; 3 - Sternum, maxillae, labium, scale $0.5 \mathrm{~mm} ; 4$ - Chelicerae, scale 1mm; 5 - Chelicerae teeth, scale $1 \mathrm{~mm} ; 6$ - Maxillae, scale $1 \mathrm{~mm} ; 7$ - Spinnerets, scale $1 \mathrm{~mm}$

Sternum (Fig. 3): 6.22 long, 5.06 wide. Oval, high in centre, sloping gradually, reddish-brown, covered with dense mat of short black hair. Posterior tip sharp but not separating coxae IV. Long black hair radiating margin.

Sigilla (Fig. 3): Three pairs, posterior, oval, 1.16 diameter, ca. 1.00 apart, 1.16 from margin, submarginal; middle, oval, 0.62 diameter, ca. 3.50 apart, 
Table 1. Haploclastus validus, measurements of legs and palp of male specimen from Matheran, WILD-10-ARA-1102. Ranges and mean include all male specimens collected from Maharashtra.

\begin{tabular}{|c|c|c|c|c|c|c|c|c|c|c|c|c|c|c|c|}
\hline & \multicolumn{3}{|c|}{ Leg I } & \multicolumn{3}{|c|}{ Leg II } & \multicolumn{3}{|c|}{ Leg III } & \multicolumn{3}{|c|}{ Leg IV } & \multicolumn{3}{|c|}{ Palp } \\
\hline & $1102^{*}$ & Range & $\begin{array}{c}\text { Mean } \pm \\
\text { SD }\end{array}$ & $1102^{*}$ & Range & $\begin{array}{c}\text { Mean } \pm \\
\text { SD }\end{array}$ & $1102^{*}$ & Range & $\begin{array}{l}\text { Mean } \pm \\
\text { SD }\end{array}$ & $1102^{*}$ & Range & $\begin{array}{c}\text { Mean } \pm \\
\text { SD }\end{array}$ & $1102^{*}$ & Range & $\begin{array}{l}\text { Mean } \pm \\
\text { SD }\end{array}$ \\
\hline Femur & 11.08 & \begin{tabular}{|c|}
$11.08-$ \\
12.39
\end{tabular} & $\begin{array}{l}11.73 \\
\pm 0.92\end{array}$ & 9.76 & $\begin{array}{l}9.76- \\
11.80\end{array}$ & $\begin{array}{r}10.78 \\
\pm 1.44\end{array}$ & 8.80 & $\begin{array}{l}8.80- \\
11.04\end{array}$ & $\begin{array}{c}9.92 \pm \\
1.58\end{array}$ & 11.10 & $\begin{array}{l}11.10- \\
12.76\end{array}$ & $\begin{array}{r}11.93 \\
\pm 1.17\end{array}$ & 6.20 & $\begin{array}{l}6.20- \\
6.10\end{array}$ & $\begin{array}{c}6.15 \pm \\
0.07\end{array}$ \\
\hline Patella & 5.38 & $\begin{array}{l}5.38- \\
6.94\end{array}$ & $\begin{array}{c}6.16 \\
\pm 1.10\end{array}$ & 5.10 & $\begin{array}{l}5.10- \\
4.98\end{array}$ & $\begin{array}{c}5.04 \pm \\
0.08\end{array}$ & 4.04 & $\begin{array}{l}4.04- \\
3.24\end{array}$ & $\begin{array}{c}3.64 \pm \\
0.56\end{array}$ & 5.04 & $\begin{array}{l}5.04- \\
3.29\end{array}$ & $\begin{array}{c}4.17 \pm \\
1.23\end{array}$ & 3.06 & $\begin{array}{l}3.06- \\
2.42\end{array}$ & $\begin{array}{c}2.74 \pm \\
0.45\end{array}$ \\
\hline Tibia & 9.08 & $\begin{array}{l}9.08- \\
7.78\end{array}$ & $\begin{array}{c}8.43 \\
\pm 0.91\end{array}$ & 7.56 & $\begin{array}{l}7.56- \\
7.60\end{array}$ & $\begin{array}{c}7.58 \pm \\
0.02\end{array}$ & 6.32 & $\begin{array}{l}6.32- \\
6.46\end{array}$ & $\begin{array}{c}6.39 \pm \\
0.09\end{array}$ & 9.26 & $\begin{array}{l}9.26- \\
9.78\end{array}$ & $\begin{array}{c}9.52 \pm \\
0.36\end{array}$ & 6.12 & $\begin{array}{l}6.12- \\
5.94\end{array}$ & $\begin{array}{l}6.03 \pm \\
0.12\end{array}$ \\
\hline Metatarsus & 7.40 & $\begin{array}{l}7.40- \\
6.72\end{array}$ & $\begin{array}{c}7.06 \\
\pm 0.47\end{array}$ & 6.32 & $\begin{array}{l}6.32- \\
5.79\end{array}$ & $\begin{array}{c}6.06 \pm \\
0.34\end{array}$ & 7.26 & $\begin{array}{l}7.26- \\
7.02\end{array}$ & $\begin{array}{l}7.14 \pm \\
0.16\end{array}$ & 10.42 & $\begin{array}{c}10.42- \\
9.80\end{array}$ & $\begin{array}{c}10.11 \\
\pm 0.43\end{array}$ & - & - & - \\
\hline Tarsus & 4.44 & $\begin{array}{c}4.44- \\
4.51\end{array}$ & $\begin{array}{c}4.47 \\
\pm 0.04\end{array}$ & 4.38 & $\begin{array}{c}4.38- \\
4.70\end{array}$ & $\begin{array}{c}4.54 \pm \\
0.01\end{array}$ & 3.72 & $\begin{array}{l}3.72- \\
4.72\end{array}$ & $\begin{array}{c}4.22 \pm \\
1.70\end{array}$ & 4.36 & $\begin{array}{c}4.36- \\
5.11\end{array}$ & $\begin{array}{c}4.74 \pm \\
0.53\end{array}$ & 2.18 & $\begin{array}{c}2.18- \\
1.50\end{array}$ & $\begin{array}{l}1.84 \pm \\
0.48\end{array}$ \\
\hline Total & 37.38 & $\begin{array}{l}37.38- \\
39.36\end{array}$ & $\begin{array}{l}38.37 \\
\pm 0.69\end{array}$ & 33.12 & $\begin{array}{l}33.12- \\
34.57\end{array}$ & $\begin{array}{r}33.85 \\
\pm 1.02\end{array}$ & 30.14 & $\begin{array}{l}30.14- \\
32.49\end{array}$ & $\begin{array}{l}31.32 \\
\pm 0.66\end{array}$ & 40.18 & $\begin{array}{c}40.18- \\
40.75\end{array}$ & $\begin{array}{c}40.47 \\
\pm 0.40\end{array}$ & 17.56 & $\begin{array}{c}17.56- \\
15.96\end{array}$ & $\begin{array}{r}16.76 \\
\pm 1.31\end{array}$ \\
\hline \multicolumn{16}{|c|}{ Midwidth } \\
\hline Femur & 3.98 & $\begin{array}{c}3.98- \\
2.9\end{array}$ & $\begin{array}{c}3.44 \\
\pm 1.12\end{array}$ & 3.34 & $\begin{array}{l}3.34- \\
2.65\end{array}$ & $\begin{array}{l}3.0 \pm \\
0.48\end{array}$ & 3.64 & $\begin{array}{l}3.64- \\
2.94\end{array}$ & $\begin{array}{c}3.29 \pm \\
0.49\end{array}$ & 3.96 & $\begin{array}{l}3.96- \\
2.45\end{array}$ & $\begin{array}{c}3.21 \pm \\
1.06\end{array}$ & 1.68 & $\begin{array}{l}1.68- \\
1.40\end{array}$ & $\begin{array}{c}1.54 \pm \\
0.19\end{array}$ \\
\hline Tibia & 2.32 & $\begin{array}{l}2.32- \\
1.90\end{array}$ & $\begin{array}{c}2.11 \\
\pm 0.29\end{array}$ & 2.06 & $\begin{array}{c}2.06- \\
1.89\end{array}$ & $\begin{array}{c}1.98 \pm \\
0.12\end{array}$ & 2.40 & $\begin{array}{c}2.40- \\
1.94\end{array}$ & $\begin{array}{c}2.17 \pm \\
0.32\end{array}$ & 2.22 & $\begin{array}{l}2.22- \\
1.91\end{array}$ & $\begin{array}{c}2.07 \pm \\
0.21\end{array}$ & 2.36 & $\begin{array}{c}2.36- \\
1.84\end{array}$ & $\begin{array}{l}2.1 \pm \\
0.36\end{array}$ \\
\hline
\end{tabular}

$1102^{*}$ - Specimen from Matheran

ca. 0.20 from margin and anterior, marginal and round.

Legs: Formula 4123. Leg III clearly thicker than the rest. Metatarsi I 1.50 times longer than tarsi I, metatarsi II 1.31 times longer than tarsi II, metatarsi III 1.50 times longer than tarsi III, metatarsi IV 1.91 times longer than tarsi IV.

Metaspines: I, 1 ventral; II, 1 ventral, 3 ventrolateral; III, 5 dorsal, 1 ventral, 6 ventrolateral; V: 1 dorsal, 1 ventral, 6 ventrolateral, 2 prolateral. Absent elsewhere.

Trichobothria: ta I, 24 clavate, 13 long 8 short filiform, ta II, 19 clavate, 12 long, 8 short filiform; ta III, 20 clavate, 12 long, 6 short filiform; IV, 15 clavate 10 long, 6 short filiform.

Leg Coxae: Coxal base dorsally easily seen from above. I longest, about 1.23 times longer than II. Coxae IV widest. Coxae I-IV covered with short and long black hair with black bristles with pallid tips; I-II sloping forward and III-IV sloping backwards.

Claws: Paired bare claws on all legs; palp with a single bare claw.

Abdomen pilosity (Fig. 1): Dorsally covered with mat of short brown hair intermixed with long black and pallid hair and ventrally with short and long brown hair with yellow cuticle exposed.

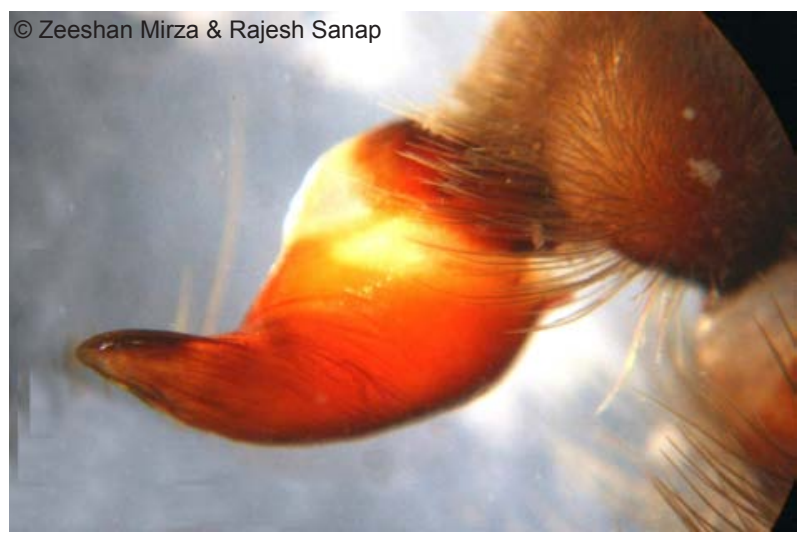

Image 2. Haploclastus validus male palpal bulb. Not to scale

Spinnerets (Fig. 7): Two pairs, yellowish brown covered with golden hair.

Male Palp (Image 2, Fig. 9-11): Embolus broad at base slightly twisted forward and flatten, flattened end resembles a duck's bill which tapers into a stout point. Ventrally with rows of poorly developed keels.

\section{Morphometry of WILD-10-ARA-543:}

Total length 26.10. Carapace 14.45 long, 9.11; chelicerae 6.10 long. Sternum: 6.22 long, 5.06 wide. Labium: 2.56 long, 2.16 wide. Abdomen 11.54 long, 

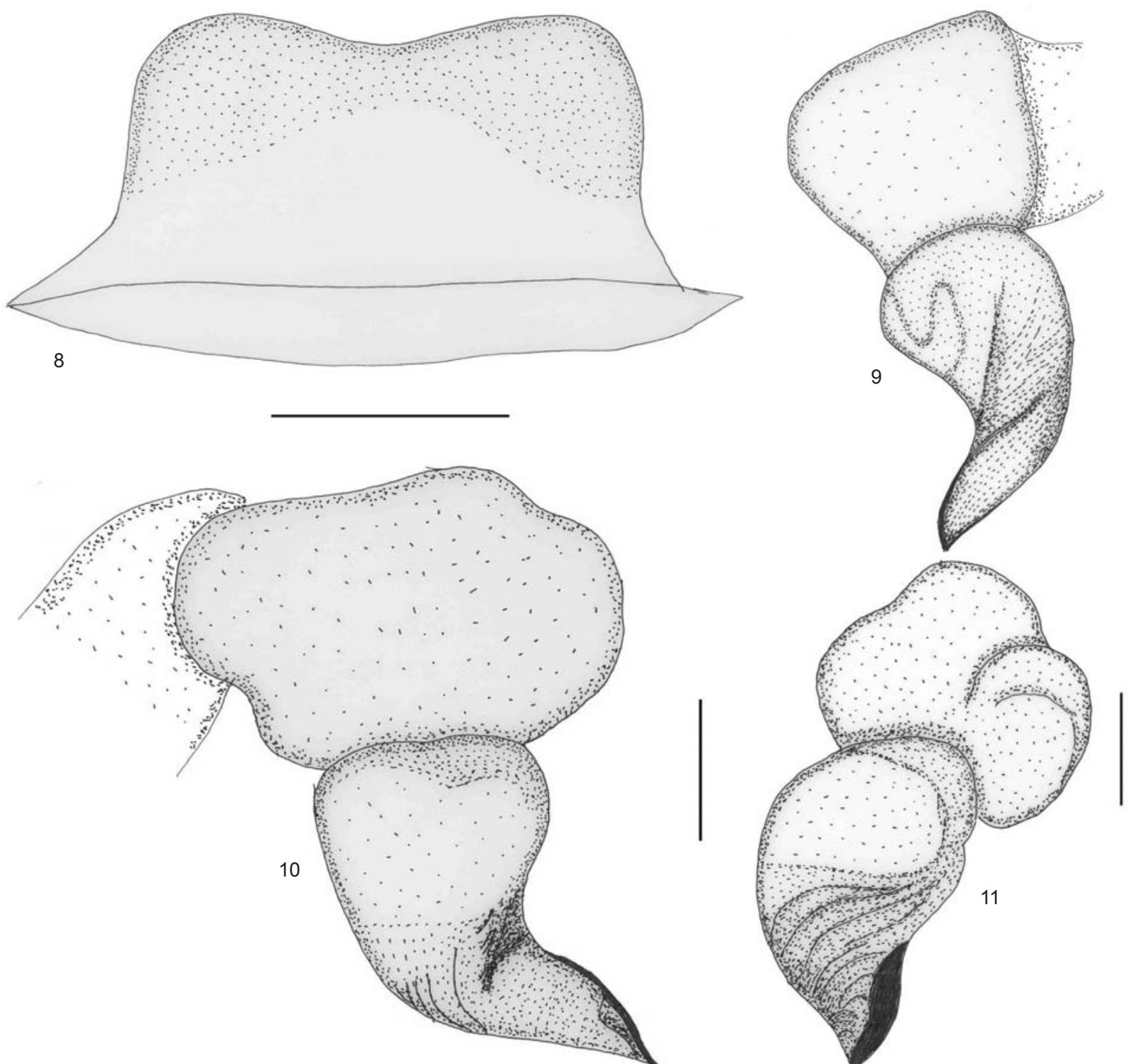

Figures 8-11. 8 - Spermathecae; 9 - Male palp, prolateral view; 10 - Male palp, retrolateral view; 11 - Male palp, ventral view (scale $1 \mathrm{~mm}$ )

8.06 wide. Spinnerets: PMS, 1.50 long, 0.58 wide, 0.60 apart; PLS, 2.08 basal, 1.76 middle, 2.02 distal; midwidths, $1.06,0.98,0.60$ respectively; 5.86 total length.

Abdomen pilosity: Cuticle not visible dorsally in juveniles and sub-adults. Large adults (especially those containing eggs in the body cavity) with cuticle entirely exposed with a fine layer of scattered short golden hair.

\section{Redescription of female, WILD-10-ARA-1103:}

Carapace 14.39 long, 12.60 wide, chelicerae 7.94. Abdomen 17.50 long, 15.02 wide. Spinnerets: PMS,
2.04 long, 0.74 wide, 0.85 apart; PLS, 3.31 basal, 1.56 middle, 2.47, distal; midwidths $1.35,1.09,0.85$, respectively and 1.30 apart. Morphometry of leg and palp given in Table 2 .

Colour in life (Image 3): Carapace reddish-brown overall with two prominent (after preservation) black bands emerging from fovea running across caput, entire surface covered with short black and golden hair. Abdomen black dorsally, yellowish on ventral and ventrolateral side covered with short and long black hair. Legs overall covered with long as well as short brown hair.

Carapace: Fovea deep, procurved. Length to 
Table 2. H. validus, measurements of legs and palp of female specimen from Matheran, WILD-10-ARA-1103. Ranges and mean include all female specimens collected from Maharashtra

\begin{tabular}{|c|c|c|c|c|c|c|c|c|c|c|c|c|c|c|c|}
\hline & \multicolumn{3}{|c|}{ Leg I } & \multicolumn{3}{|c|}{ Leg II } & \multicolumn{3}{|c|}{ Leg III } & \multicolumn{3}{|c|}{ Leg IV } & \multicolumn{3}{|c|}{ Palp } \\
\hline & $1103^{*}$ & Range & $\begin{array}{c}\text { Mean } \pm \\
\text { SD }\end{array}$ & $1103^{*}$ & Range & $\underset{\text { Mean } \pm}{\text { SD }}$ & $1103^{*}$ & Range & $\begin{array}{c}\text { Mean } \pm \\
\text { SD }\end{array}$ & $1103^{*}$ & Range & $\begin{array}{l}\text { Mean } \pm \\
\text { SD }\end{array}$ & $1103^{*}$ & Range & $\begin{array}{c}\text { Mean } \pm \\
\text { SD }\end{array}$ \\
\hline Femur & 5.68 & $\begin{array}{l}5.68- \\
9.48\end{array}$ & $\begin{array}{c}7.58 \pm \\
2.69\end{array}$ & 5.36 & $\begin{array}{l}5.36- \\
8.29\end{array}$ & $\begin{array}{c}6.83 \pm \\
2.07\end{array}$ & 4.78 & $\begin{array}{l}4.78- \\
7.30\end{array}$ & $\begin{array}{c}6.04 \pm \\
1.78\end{array}$ & 7.06 & $\begin{array}{c}7.06- \\
9.68\end{array}$ & $\begin{array}{c}8.37 \pm \\
1.85\end{array}$ & 4.68 & $\begin{array}{l}4.68- \\
6.68\end{array}$ & $\begin{array}{c}5.68 \pm \\
1.41\end{array}$ \\
\hline Patella & 4.26 & $\begin{array}{c}4.26- \\
5.69\end{array}$ & $\begin{array}{c}4.98 \pm \\
1.01\end{array}$ & 3.58 & $\begin{array}{l}3.58- \\
7.08\end{array}$ & $\begin{array}{c}5.33 \pm \\
2.47\end{array}$ & 3.56 & $\begin{array}{l}3.56- \\
5.42\end{array}$ & $\begin{array}{c}4.49 \pm \\
1.31\end{array}$ & 4.10 & $\begin{array}{c}4.10- \\
6.45\end{array}$ & $\begin{array}{c}5.28 \pm \\
1.66\end{array}$ & 2.72 & $\begin{array}{l}2.72- \\
3.32\end{array}$ & $\begin{array}{c}3.02 \pm \\
0.42\end{array}$ \\
\hline Tibia & 4.82 & $\begin{array}{l}4.82- \\
6.61\end{array}$ & $\begin{array}{c}5.72 \pm \\
1.26\end{array}$ & 2.94 & $\begin{array}{l}2.94- \\
5.21\end{array}$ & $\begin{array}{c}4.08 \pm \\
1.60\end{array}$ & 2.68 & $\begin{array}{l}2.68- \\
4.04\end{array}$ & $\begin{array}{c}3.36 \pm \\
0.96\end{array}$ & 4.42 & $\begin{array}{l}4.42- \\
6.74\end{array}$ & $\begin{array}{c}5.58 \pm \\
1.64\end{array}$ & 2.80 & $\begin{array}{l}2.80- \\
4.18\end{array}$ & $\begin{array}{c}3.49 \pm \\
0.97\end{array}$ \\
\hline Metatarsus & 2.22 & $\begin{array}{c}2.22- \\
4.11\end{array}$ & $\begin{array}{c}3.17 \pm \\
1.32\end{array}$ & 2.18 & $\begin{array}{c}2.18- \\
4.08\end{array}$ & $\begin{array}{c}3.13 \pm \\
1.34\end{array}$ & 3.24 & $\begin{array}{l}3.24- \\
4.73\end{array}$ & $\begin{array}{c}3.99 \pm \\
1.05\end{array}$ & 5.02 & $\begin{array}{l}5.02- \\
7.58\end{array}$ & $\begin{array}{l}6.3 \pm \\
1.81\end{array}$ & - & - & - \\
\hline Tarsus & 1.70 & $\begin{array}{l}1.70- \\
3.77\end{array}$ & $\begin{array}{c}2.74 \pm \\
1.46\end{array}$ & 2.04 & $\begin{array}{l}2.04- \\
3.34\end{array}$ & $\begin{array}{c}2.69 \pm \\
0.91\end{array}$ & 1.88 & $\begin{array}{l}1.88- \\
3.49\end{array}$ & $\begin{array}{c}2.69 \pm \\
1.13\end{array}$ & 2.18 & $\begin{array}{l}2.18- \\
3.64\end{array}$ & $\begin{array}{c}2.91 \pm \\
1.03\end{array}$ & 2.88 & $\begin{array}{c}2.88- \\
4.16\end{array}$ & $\begin{array}{c}3.52 \pm \\
0.90\end{array}$ \\
\hline Total & 18.68 & $\begin{array}{l}\text { 18.68- } \\
29.66\end{array}$ & $\begin{array}{c}24.17 \pm \\
7.76\end{array}$ & 16.1 & $\begin{array}{c}16.1- \\
28\end{array}$ & $\begin{array}{c}22.05 \pm \\
8.41\end{array}$ & 16.14 & $\begin{array}{l}16.14- \\
24.98\end{array}$ & $\begin{array}{r}20.56 \\
\pm 6.25\end{array}$ & 22.78 & $\begin{array}{c}22.78- \\
34.09\end{array}$ & $\begin{array}{l}28.44 \\
\pm 7.99\end{array}$ & 13.08 & $\begin{array}{l}13.08- \\
18.34\end{array}$ & $\begin{array}{r}15.71 \\
\pm 3.71\end{array}$ \\
\hline \multicolumn{16}{|c|}{ Midwidth } \\
\hline Femur & 1.70 & $\begin{array}{l}1.70- \\
2.70\end{array}$ & $\begin{array}{l}2.2 \pm \\
0.70\end{array}$ & 1.88 & $\begin{array}{l}1.88- \\
2.48\end{array}$ & $\begin{array}{c}2.18 \pm \\
0.42\end{array}$ & 2.40 & $\begin{array}{l}2.40- \\
3.32\end{array}$ & $\begin{array}{c}2.86 \pm \\
0.65\end{array}$ & 1.86 & $\begin{array}{l}1.86- \\
2.71\end{array}$ & $\begin{array}{c}2.29 \pm \\
0.60\end{array}$ & 1.16 & $\begin{array}{l}1.16- \\
1.68\end{array}$ & $\begin{array}{c}1.92 \pm \\
0.36\end{array}$ \\
\hline Tibia & 1.80 & $\begin{array}{l}1.80- \\
2.85\end{array}$ & $\begin{array}{c}2.33 \pm \\
0.74\end{array}$ & 1.78 & $\begin{array}{l}1.78- \\
2.64\end{array}$ & $\begin{array}{c}2.21 \pm \\
0.60\end{array}$ & 1.90 & $\begin{array}{l}1.90- \\
2.59\end{array}$ & $\begin{array}{c}2.25 \pm \\
0.48\end{array}$ & 1.92 & $\begin{array}{l}1.92- \\
2.74\end{array}$ & $\begin{array}{c}2.33 \pm \\
0.38\end{array}$ & 1.42 & $\begin{array}{l}1.42- \\
2.06\end{array}$ & $\begin{array}{l}1.74 \pm \\
0.42\end{array}$ \\
\hline
\end{tabular}

$1103^{*}$ - Specimen from Matheran

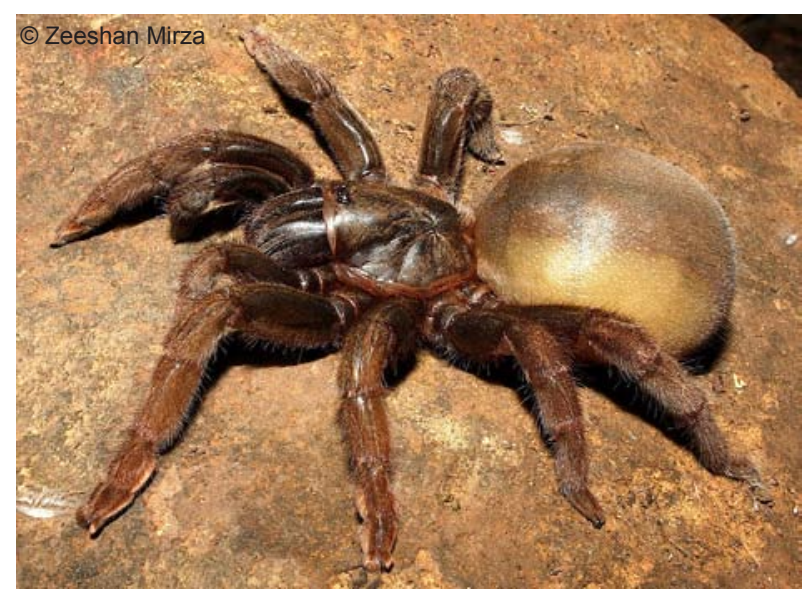

Image 3. Haploclastus validus female from Aarey Milk Colony (Mumbai, Maharashtra) depicting coloration in life. Not collected

width ratio 1.14. Caput raised high and eye group on a tubercle. Bristles: 11 long on caput in mid-dorsal line; 4 long, 16 short antero-medially; 6 long, 18 short between PME; 10 long, 13 short on clypeus edge.

Eyes: Ratio of eye group width to length 2.4. ALE clearly larger than rest, AME slightly larger than PLE, PLE and PME almost equal in diameter. ALE 0.58, AME 0.47, PLE 0.32, PME 0.31; ALE-AME 0.23, AME-AME 0.40, PLE-PME 0.11, PME-PME 1.06, ALE-PLE 0.30, PLE-PLE 1.79, ALE-ALE 1.50. Ocular group 0.80 long and 2.34 wide. MOQ 0.68

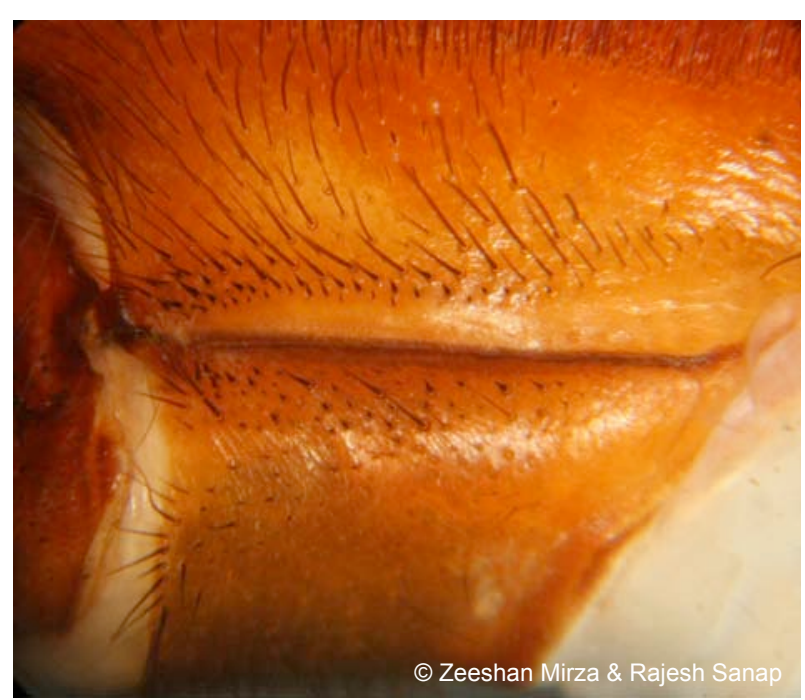

Image 4. Haploclastus validus female maxillae prolateral view. Not in scale

long; front width 0.90 and back width 1.18. Clypeus absent.

Maxillae: Prolateral face with three to four rows of thorn setae intermixed with long spine-like bristles above and below the maxillary suture. Long spine setae scattered above as well as below the thorn rows. Cuspules ca. 200 in a triangular patch in the anterior corner.

Maxillary lyra (Image 4): No definite shape, small 
thorn setae above and below suture arranged randomly. No ventral brush of lyra.

Labium: 2.56 long, 2.16 wide, labiosternal grove shallow, 265 cuspules similar in size to those on the maxilla.

Chelicerae: Ectal lyrate, grove glaborous; lyra, straight, black spines in 3-4 rows. Promarginal teeth in 2 rows 15 outer large teeth, 10 small on inner edge adjacent to the larger teeth; 49 basomesal teeth in 3-4 rows.

Sternum: 7.91 long and 5.72 wide. Longer than wide, high at $1 / 3$ region and gradually sloping. Posterior edge acutely sharp, not separating coxae IV. Sternum reddish-brown with a mat of golden short hairs. Long black hair radiating from the marginal area.

Sigilla: Three pairs, posterior, oval, 1.19 diameter, ca. 1.04 apart, 1.7 from margin; middle, oval, 0.79 diameter, ca. 3.43 apart,0.04 from margin and anterior, marginal and round.

Legs: Formula 4123. Leg III clearly thicker than the rest. Metatarsi I 1.09 times longer than tarsi I, metatarsi II 1.22 times longer than tarsi II, metatarsi III 1.40 times longer than tarsi III, metatarsi IV 2.08 times longer than tarsi IV.

Metaspines: I: 1 ventral; II: 2 ventrolateral; III: 2 ventral, 6 ventrolateral, 2 dorsal; V: 1dorsal, 2 ventral, 6 ventrolateral.Absent elsewhere.

Trichobothria: ta I, 26 clavate, 12 long 4 short filiform, ta II, 22 clavate, 12 long, 7 short filiform; ta III, 21 clavate, 10 long, 5 short filiform; IV, 17 clavatem 10 long, 6 short filiform. Clavate in distal half in middorsal, filiform in distal $2 / 3^{\text {rd }}$ in two rows.

Leg coxae: Numerous short scattered spinules on prolateral face of coxae of leg I, II, III and IV. Leg I-III with median narrow light brush, IV glabrous. II-IV with setose mound up from inner corner low mound with few bristles. All retrolaterally lack ventral edge and ventrally with uniform setation. Coxal base dorsally easily seen from above. I longest, about 1.11 times longer than II. Coxae IV widest. Coxae I-IV covered with a mat of golden hair and also with long black hair; I-II sloping forward and III-IV sloping backwards.

Claws: Paired bare claws on all legs; palp with a single bare claw.

Abdomen pilosity: Dorsally covered with a mat of short brown hair intermixed with long black and pallid hair and ventrally with short and long brown hair with yellow cuticle exposed.

Spinnerets: Two pairs, yellowish-brown covered with golden hair.

Spermathecae (Fig. 8): Fused seminal receptacle. A brick-shaped structure with upper edges smooth and rounded, present to form small mounds.

\section{Variations:}

Total length 16.11-31.89 (23.14 \pm 8.03$)$. Carapaces 7.28-14.39 (9.97 \pm 3.86$)$ long, 6.02-12.6 (8.81 \pm 3.4$)$ wide. Sternum: 3.56-7.91 (5.36 \pm 2.27) long, 3.075.72 (4.32 \pm 1.33$)$ wide. Labium: 0.98-2.16 (1.59 \pm 0.59$)$ long, 1.3-2.56 (1.8 \pm 0.67$)$ wide. Abdomen range 8.83-

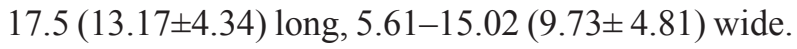
Spinnerets: PMS, 1.18-2.04 (0.60 \pm SD 0.60) long, $0.70-0.74$ (mean \pm SD 0.03) wide, $0.48-0.85$ (mean \pm SD 0.26) apart; PLS, 1.28-3.31 (2.07士 SD 1.09) basal, $1.05-1.56(1.26 \pm 0.27)$ middle, $0.51-2.47$ (1.46 \pm 0.98$)$ distal; midwidths, 0.98-1.35 (1.19 \pm 0.19$), 0.89-1.09$ $(0.96 \pm 0.11), \quad 0.70-0.85 \quad(0.75 \pm 0.08)$ respectively; 2.84-7.34 (4.79 \pm 2.31$)$ total length.

Abdomen pilosity: Cuticle not visible dorsally in juveniles and sub-adults. Large adults (especially those containing eggs in the body cavity) with cuticle entirely exposed with a fine layer of scattered short golden hair.

\section{Distribution}

India (Image 5): Maharashtra: Matheran, Raighad District; Jauli, Satara District; Aarey Milk Colony, Sanjay Gandhi National Park, Mumbai; Kolad, Raighad District; Bhimashankar Wildlife Sanctuary, Pune District.

\section{NATURAL HISTORY}

Matheran is situated at an elevation of $800 \mathrm{~m}$ and the forest is of semi-evergreen type. The forest in this area is contiguous to that of Prabalgadh and Bhimashankar Wildlife Sanctuary. Terminalia arjuna, Mallotus philippensis, Albizia amara, Ficus racemosa, Atalantia racemosa, Carvia callosa, Olea dioica, Mangifera indica, Pittosporum dasycaulon and Memycylon umbellatum (Image 6) dominate this area. The temperature fluctuates between $13-34{ }^{\circ} \mathrm{C}$ and the highest rainfall recorded is $850 \mathrm{~mm}$. Male 


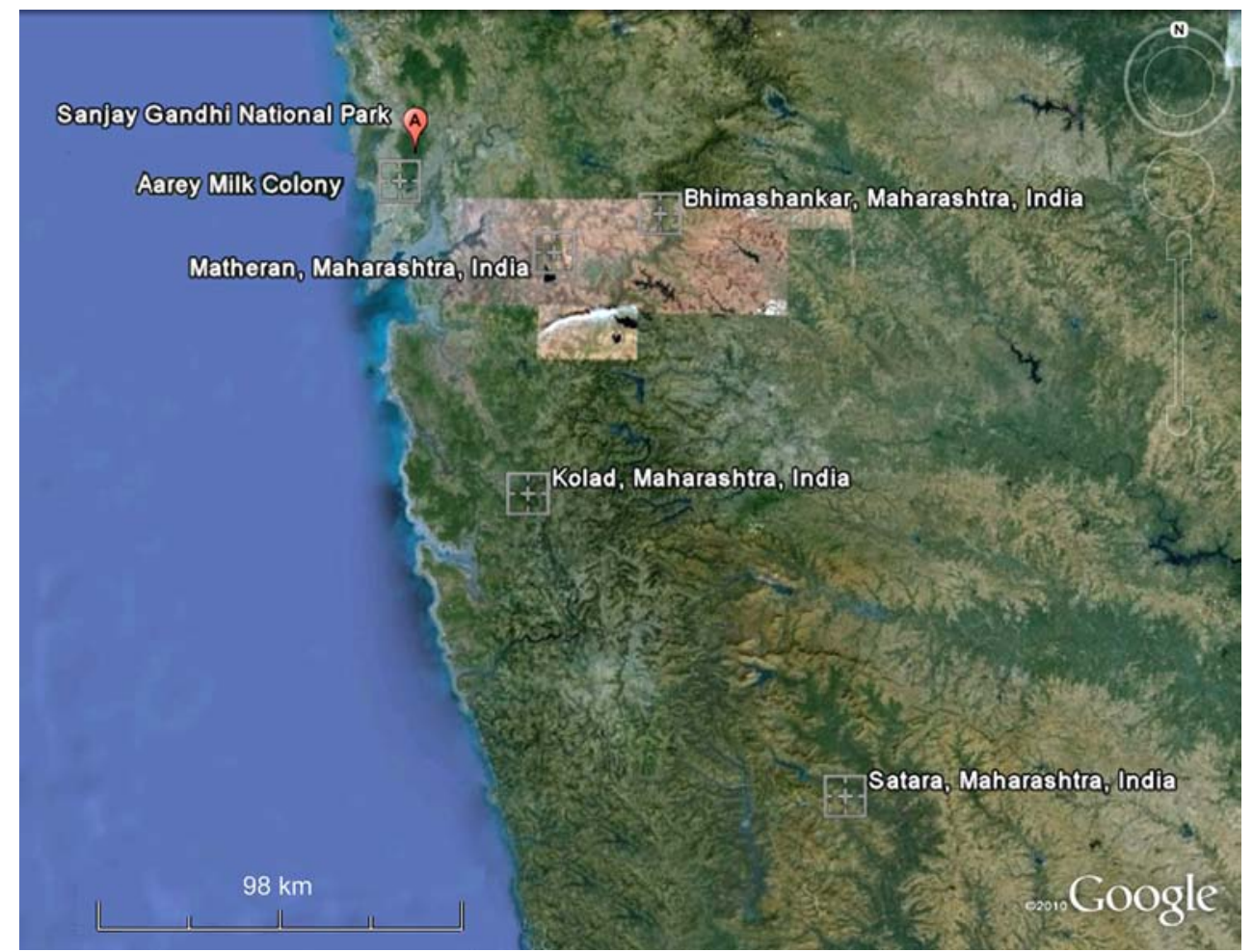

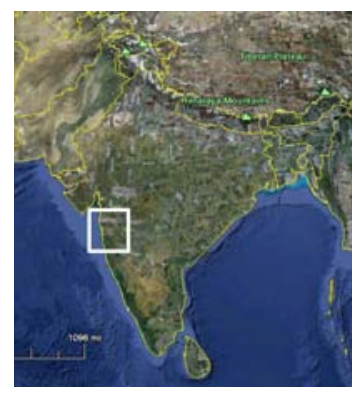

Image 5. The northern Western Ghats of Maharashtra showing the known range of Haploclastus validus

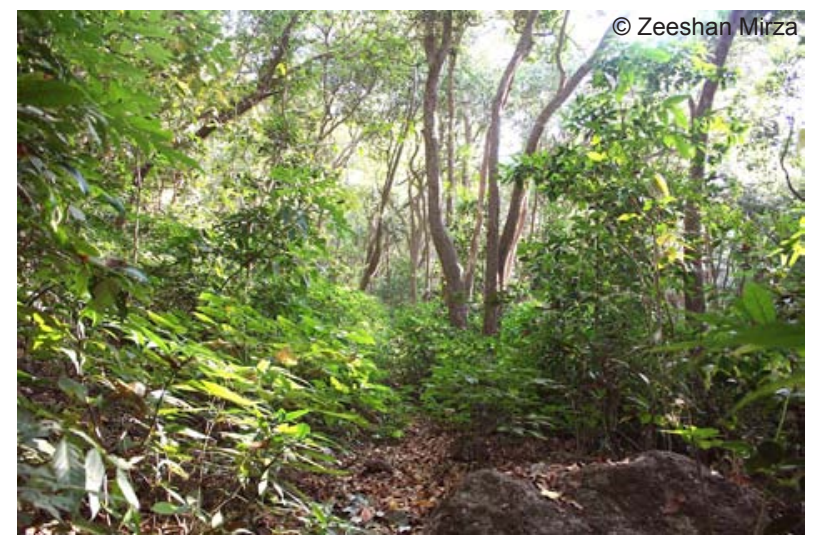

Image 6. Collection site at Matheran (Raighad District, Maharashtra)

specimens of $H$. validus were commonly encountered during night walks from June to October. A single female was found after excavating a burrow found under a rotting $\log$ in a private estate. The habitat at Aarey Milk Colony is highly degraded and altered. The vegetation in the area is of deciduous type and heavily mixed with exotic species. Some of these include Butea monosperma, Tectona grandis, Acacia spp., Ziziphus spp., Pongamia pinnata, Cassia fistula, Mangifera indica, Gliricidia sepium, Delonix regia and Eucalyptus melliodora.
Most of the burrows were found at the base of trees and some in the vicinity of large boulders. All burrows had double door entrances leading to a single tube except for the female found in Bhimashankar, that burrow had a single entrance. The door is made up of a thin layer of silk to which the surrounding soil adheres making the outer layer indistinguishable from its surroundings. The door is usually ' $\mathrm{D}$ ' shaped or circular, ranging from 10 to $23.62 \mathrm{~mm}$ in diameter and 1.80 to $4 \mathrm{~mm}$ thick (Image 7). The doors are hinged to the burrow on one side. The burrow is covered with a fine layer of silk. The entrance of the burrow of one specimen from Aarey measured $17.74 \mathrm{~mm}$. The burrows are 12 inches to 24 inches deep. The burrow end is slightly wider than the entrance forming a chamber. More than $8-10$ burrows can be found at the base of a single tree.

The male from Aarey Milk Colony was found under a rotten tree trunk during the monsoons and the one from Matheran was dug out from its burrow in the month of April along with its exuvia. Both the females were dug out from their burrows. Several burrows at Aarey Milk Colony were excavated. One of the excavated burrows contained a female carrying an egg sac below her chelicerae (Image 8) and the other burrows had only the egg sac silk cover from which juveniles 


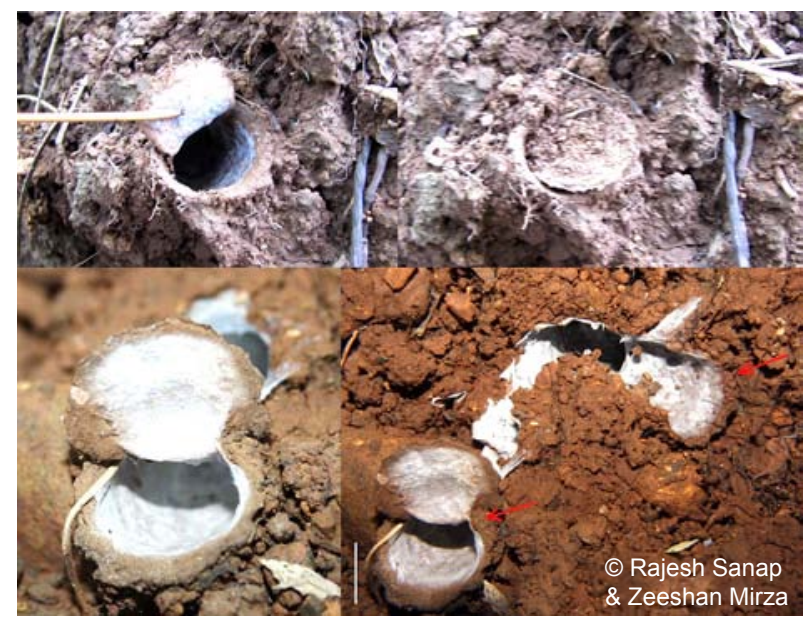

Image 7. Trapdoor burrow, depicting the structure and shape of the door and the entrance

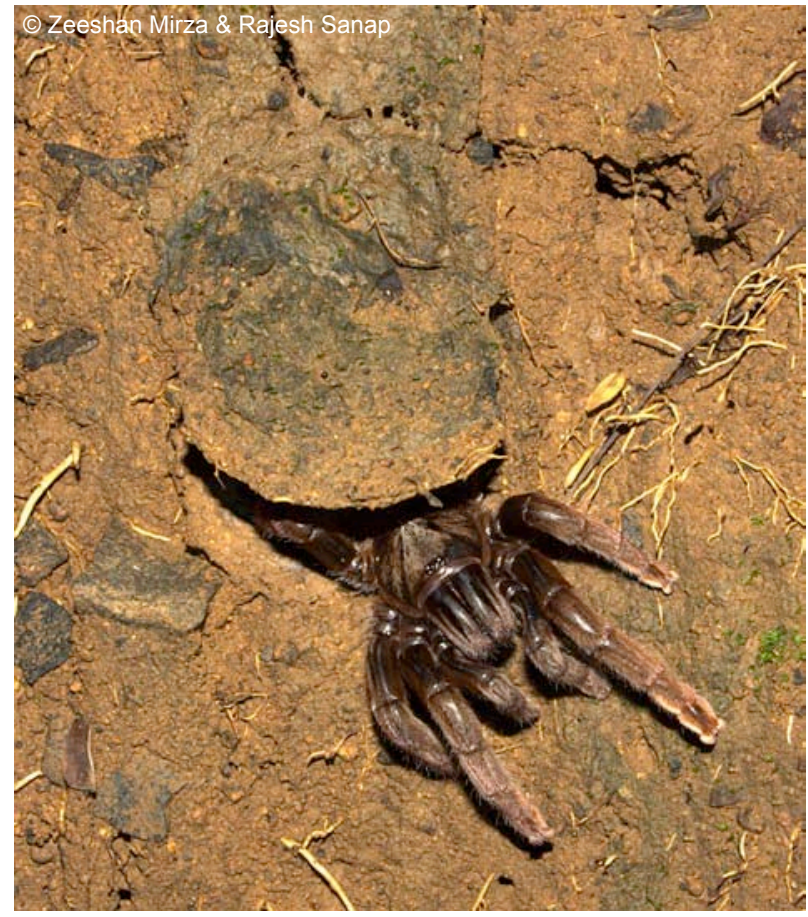

Image 9. Female Haploclastus validus at the entrance of the burrow. Not collected

had already dispersed. A burrow was found with the entrance covered with a thick layer of web covering the entrance behind the door in the month of March. The burrow was observed till the first week of June when the web covering was absent and a few juveniles were observed at the periphery of the entrance inside the burrow (Image 9). This indicates that the female mates during the monsoon and post monsoon periods and lays eggs by late winter or early summer and the

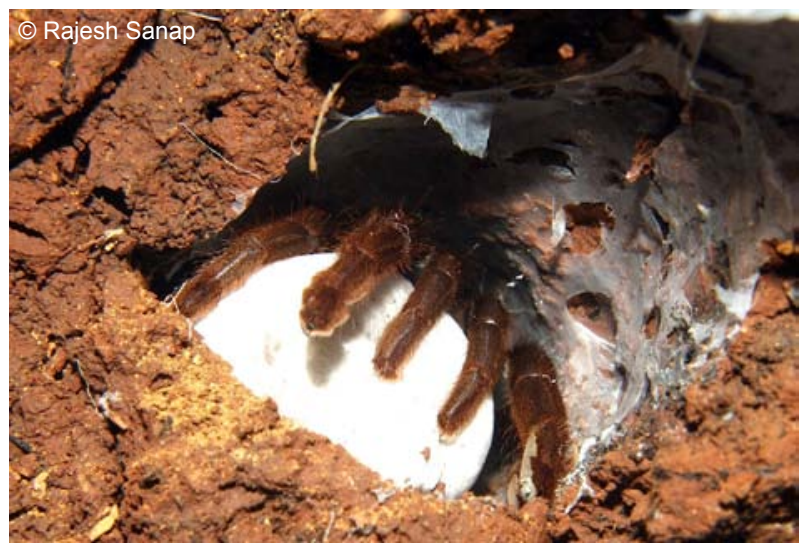

Image 8. Female Haploclastus validus with an egg sac. Not collected

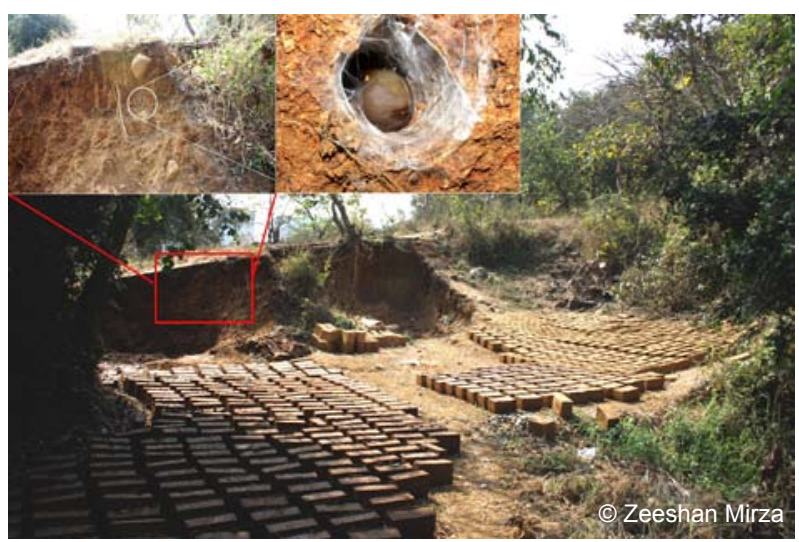

Image 10. Habitat destruction at Aarey Milk Colony for removal of soil for brick making. Note the exposed burrow due to this practice in the inset

juveniles disperse before the next monsoons. With the onset of the monsoons, males of this species can be commonly seen on roads and they die in large numbers due to the heavy vehicular traffic in Aarey Milk Colony in Mumbai and Kolad in Raighad District and are killed if they enter houses. At Aarey Milk Colony, this species has been seen in sympatry with other spiders such as Idiops bombayensis, Plesiophrictus millardi and Chilobrachys fimbriatus. This species and others are under threat at Matheran, Kolad and Aarey Milk Colony from soil erosion (Image 10), destruction of forests and from vehicular traffic. Anthropogenic activities like removal of soil leads to major loss of this species. 


\section{REFERENCES}

Dippenaar-Schoeman, A.S. (2002). Baboon and Trapdoor Spiders of Southern Africa: An Introduction Manual. Plant Protection Research Institute Handbook No. 13, Agricultural Research Council, Pretoria, 128pp.

Gravely, F.H. (1915). Notes on Indian mygalomorph Spiders. Records of Indian Museum, Calcutta 11: 257-287.

Gravely, F.H. (1935). Notes on Indian mygalomorph spiders. II. Records of Indian Museum, Calcutta 37: 69-84.

Molur, S. \& M. Siliwal (2004). Common names of South Asian Theraphosid spiders (Araneae: Theraphosidae). Zoos' Print Journal 19(10): 1657-1662.

Platnick, N.I. (2011). The World Spider Catalog, version 12.0. American Museum of Natural History, online at http://research.amnh.org/iz/spiders/catalog. DOI: 10.5531/db.iz.0001.

Pocock, R.I. (1899). Diagnoses of some new Indian Arachnida. Journal of the Bombay Natural History Society, 12: 744-753.

Pocock, R.I. (1900). The Fauna of British India, Including Ceylon and Burma. Arachnida. Taylor and Francis, London. 279pp.

Raven, R.J. (1985). The spider infraorder Mygalomorphae (Araneae): cladistics and systematics. Bulletin of the American Museum of Natural History, 182: 1-180.

Siliwal, M. \& S. Molur (2007). Checklist of spiders (Arachnida: Araneae) of South Asia including the 2006 update of Indian spider checklist. Zoos' Print Journal, 22(2): 2551-2597.

Siliwal, M. \& S. Molur (2009). Redescription, distribution and status of the Karwar Large Burrowing Spider Thrigmopoeus truculentus Pocock, 1899 (Araneae: Theraphosidae), a Western Ghats endemic ground mygalomorph. Journal of Threatened Taxa 1(6): 331-339.

Siliwal M. \& R.J. Raven (2010). Taxonomic change of two species in the genus Haploclastus Simon 1892 (Araneae, Theraphosidae). ZooKeys 46: 71-75.

Siliwal, M., S. Molur\& B.K. Biswas (2005). Indian spiders (Arachnida, Araneae): updated checklist 2005. Zoos'Print Journal 20(10): 1999-2049.

Simon, E. (1892). Histoire naturelle des araignées. Volume 1, part 1. Paris, 256pp.

Smith, A.M. (1987). The Tarantula: Classification and Identification Guide (second edition). Fitzgerald Publishing, London, 178pp.
Author Details: ZeEshan A. Mirza is a student currently perusing a bachelor's degree in science. He is interested in the study of mygalomorph spider, scorpions and reptiles. $\mathrm{He}$ is currently working on the biodiversity documentation of Aarey Milk Colony. He has described several new species of scorpions and trapdoor spiders.

RAJESH V SANAP is a graduate student interested in the study of mygalomorph spiders and scorpions. He is currently working on the biodiversity documentation of Aarey Milk Colony. He has described several new species of scorpions and trapdoor spiders.

MANJU SILIWAL is an arachnologist and currently is working as Research Associate at Wildlife Information Liaison Development Society, Coimbatore. Her expertise is on mygalomorph (primitive) spiders and has described many new species of spiders including trapdoor spiders and tarantulas.

Acknowledegments: Authors (ZM \& RS) wish to thank Vishal Shah, Shardul Bajikar, Amit Panariya, Dr. Santosh Tunagre, Vishwanath Rathode for all their help and encouragement. The Dairy Ministry is thanked for encouragements and permission to carry out surveys at Aarey Milk Colony. Agarwal Janseva Charitable Trust is thanked for providing logistic support to ZM and RS. Ashish Jadhav is thanked for assistance during field work. Special thanks to Bhavan's College for constant support and encouragement to ZM. ZM thanks Gavin Desouza and Kunal Ullalkar for their continued support and kind help. MS would like to thank DEFRA / FFI Flagship Species Fund (project No. 06/16/02 FLAG) for the financial support to the Indian Tarantula Project, during one of the survey trips this trapdoor spider was found. MS is also grateful to the following personnel: Sally Walker, Zoo Outreach Organisation (ZOO), Coimbatore for her encouragement to the spider study; Sanjay Molur, WILD/ZOO for initiating the tarantula project and Robert Raven, Queensland Museum, Australia for providing pictures of the type specimen of $H$. robustus. We wish to acknowledge Mandar Kulkarni for assistance during field work and all his help. 